\title{
Evaluación de déficit de atención con hiperactividad: la escala SNAP IV adaptada a la Argentina
}

\author{
Nora Grañana, ${ }^{1,2}$ Alba Richaudeau, ${ }^{2}$ Carlos Robles Gorriti, ${ }^{2}$ \\ Martín O'Flaherty, ${ }^{2}$ María Elena Scotti, ${ }^{3}$ Lucila Sixto, $^{3}$ \\ Ricardo Allegri ${ }^{1}$ y Natalio Fejerman ${ }^{4}$
}

Forma de citar

Grañana N, Richaudeau A, Robles Gorriti C, O’Flaherty M, Scotti ME, Sixto L, et al. Evaluación de déficit de atención con hiperactividad: la escala SNAP IV adaptada a la Argentina. Rev Panam Salud Publica. 2011;29(5):344-9.

RESUMEN Objetivo. Valorar la utilidad de la escala SNAP IV como instrumento de detección de trastorno por déficit de atención con hiperactividad (TDAH) en niños argentinos de 4 a 14 años de edad.

Métodos. Se adaptó y se administró la escala SNAP IV a un grupo de 1230 escolares de la provincia de Buenos Aires, Argentina. Se determinó el diagnóstico con el control clínico de acuerdo a los criterios del Manual diagnóstico y estadístico de los trastornos mentales, $4^{a}{ }^{a}$ edición. Se determinaron la sensibilidad y especificidad así como los puntajes de corte para la escala SNAP IV en la población estudiada.

Resultados. Se estableció el puntaje en la escala SNAP IV que tuviera la mejor correlación entre sensibilidad y especificidad para determinar los casos verdaderos positivos que realmente tuvieran un diagnóstico clínico. Los puntajes de corte obtenidos fueron: un indice de 1,66 (15/27 puntos) para la subescala déficit de atención y de 1,77 (16/27 puntos) para hiperactividadimpulsividad en la población estudiada.

Conclusiones. La escala SNAP IV para detección de TDAH se considera válida en el caso de la población estudiada, siempre y cuando se modifiquen los puntajes de corte para obtener la mejor relación sensibilidad/especificidad, con base en las particularidades culturales y socioeconómicas de dicha población.

Palabras clave Trastorno por déficit de atención con hiperactividad; salud mental; Argentina.

El trastorno por déficit de atención con hiperactividad e impulsividad se caracteriza por dificultades para prestar aten-

\footnotetext{
Laboratorio de Memoria, Hospital A. Zubizarreta, Buenos Aires, Argentina. La correspondencia se debe dirigir a Nora Grañana. Correo electrónico: ngranana@hotmail.com

2 Departamento de Pediatría, Hospital Universitario Austral, Buenos Aires, Argentina.

3 Centro de Psicopatología Infanto Juvenil, Buenos Aires, Argentina.

4 Hospital de Pediatría Juan Garrahan, Buenos Aires, Argentina.
}

ción que comienzan durante el desarrollo y conductas de hiperactividad e impulsividad que ocurren en dos o más ambientes e interfieren en la inclusión familiar, escolar y social (1). En 1902, Still describió a un grupo de niños con déficit en la atención sostenida y lo relacionó con una dificultad en el control moral de la conducta. Desde entonces ha habido muchas definiciones de atención y discusiones en torno a cómo se deben medir las dificultades atencionales y la presen- cia de conductas de hiperactividad e impulsividad durante el desarrollo.

Luego de que en los años veinte se describieran casos de este trastorno originados a raíz de la encefalitis epidémica de Von Economo, se comenzaron a sospechar sus bases neurobiológicas. Estos informes hablaron de la llamada "disfunción cerebral mínima", donde si bien el compromiso orgánico no era detectado por los estudios neurológicos de rutina, los síntomas interferían en la cali- 
dad de vida. La disponibilidad creciente de conocimientos llevó a los profesionales a interpretar el déficit atencional con hiperactividad e impulsividad como un trastorno del desarrollo, investigándose sus bases neurobiológicas, la frecuente predisposición hereditaria y su evolución crónica con impacto negativo en la inclusión tanto académica como social de los niños afectados (2). El modelo neuropsicológico de Posner describió los subcomponentes del sistema atencional, denominados atención sostenida, selectiva y control atencional. A partir del desarrollo del marco teórico, un aspecto importante es cómo se valoran estos síntomas en el contexto clínico de cada paciente y en una población determinada.

El Manual diagnóstico y estadístico de los trastornos mentales (DSM, por sus siglas en inglés), versión IV, ha considerado un grupo de 18 síntomas clínicos para definir el trastorno por déficit de atención con hiperactividad (TDAH), dividiéndolos en dos dominios: déficit de atención por un lado e hiperactividad-impulsividad por el otro (1). Para el diagnóstico de TDAH se requiere tener de seis a nueve síntomas - en uno o ambos dominiosen al menos dos ambientes habituales de funcionamiento del individuo, como por ejemplo la casa y la escuela. Así, se consideran tres subtipos de TDAH: i) con predominio de déficit de atención, ii) con predominio de hiperactividadimpulsividad, y iii) mixto.

En diversos estudios se ha tratado de demostrar qué alteraciones del sistema atencional se producen en las distintas formas de TDAH (3). Mediante las escalas de detección es posible valorar las conductas de hiperactividad, impulsividad y los síntomas de déficit de atención, así como cuantificar la severidad de los síntomas y evaluar la respuesta a tratamientos. La escala más utilizada es la de Conners, que puntúa la severidad de los síntomas con grados de cero (ninguna) a tres (mucha) (4). Hay además una versión corta, pero que es menos sensible particularmente al déficit atencional (5).

Se usa asimismo el cuestionario de Swanson, Nolan y Pelham de 1983 en su versión IV (SNAP IV), que también puntúa los síntomas de cero a tres y tiene dos versiones - para padres y para maestros- con los criterios del DSM IV (6-10). En el SNAP IV se consideran nueve preguntas para la subescala de atención y nueve para la de hiperactividadimpulsividad, cada una de las cuales con

CUADRO 1. Cuestionario SNAP IV a administrado a padres y maestros en dos escuelas del Gran Buenos Aires, para detectar trastornos por déficit de atención con hiperactividad (TDAH), Argentina, 2003

\begin{tabular}{|c|c|c|c|c|c|}
\hline & \multirow[b]{2}{*}{ Pregunta } & \multicolumn{4}{|c|}{ Puntaje } \\
\hline & & $\begin{array}{l}\text { Nada } \\
(0)\end{array}$ & $\begin{array}{l}\text { Poco } \\
(1)\end{array}$ & $\begin{array}{l}\text { Bastante } \\
\quad(2)\end{array}$ & $\begin{array}{l}\text { Mucho } \\
\text { (3) }\end{array}$ \\
\hline \multicolumn{6}{|c|}{ A menudo } \\
\hline 1 & $\begin{array}{l}\text { Le cuesta prestar atención a detalles o comete errores } \\
\text { por descuido en las tareas escolares o trabajo. }\end{array}$ & & & & \\
\hline 2 & $\begin{array}{l}\text { Tiene dificultades para mantener la atención en tareas } \\
\text { o en actividades lúdicas. }\end{array}$ & & & & \\
\hline 3 & Parece no escuchar cuando se le habla directamente. & & & & \\
\hline 4 & $\begin{array}{l}\text { Le cuesta seguir instrucciones y no finaliza tareas } \\
\text { escolares, encargos u obligaciones. }\end{array}$ & & & & \\
\hline 5 & Tiene dificultad en organizar sus tareas y actividades. & & & & \\
\hline 6 & $\begin{array}{l}\text { Evita, le disgusta o es reacio a dedicarse a tareas que } \\
\text { requieren un esfuerzo mental sostenido. }\end{array}$ & & & & \\
\hline 7 & $\begin{array}{l}\text { Extravía objetos necesarios para realizar sus actividades } \\
\text { (p. ej. juguetes, ejercicios escolares, lápices o libros). }\end{array}$ & & & & \\
\hline 8 & Se distrae por estímulos irrelevantes de su tarea. & & & & \\
\hline 9 & Es descuidado en sus actividades diarias. & & & & \\
\hline 10 & $\begin{array}{l}\text { Le cuesta mantenerse alerta, responder a lo que se le } \\
\text { pide, o ejecutar consignas. }\end{array}$ & & & & \\
\hline 11 & Mueve las manos y los pies o se retuerce en el asiento. & & & & \\
\hline 12 & $\begin{array}{l}\text { Abandona su asiento en clase u otras situaciones en } \\
\text { que se espera que permanezca sentado. }\end{array}$ & & & & \\
\hline 13 & $\begin{array}{l}\text { Corre o salta excesivamente en situaciones en que es } \\
\text { inapropiado. }\end{array}$ & & & & \\
\hline 14 & $\begin{array}{l}\text { Tiene dificultades para jugar o dedicarse a actividades } \\
\text { de ocio tranquilamente. }\end{array}$ & & & & \\
\hline 15 & $\begin{array}{l}\text { Está "en marcha" o actúa como si tuviera un motor } \\
\text { encendido. }\end{array}$ & & & & \\
\hline 16 & Habla en exceso. & & & & \\
\hline 17 & $\begin{array}{l}\text { Precipita respuestas antes de haber sido terminadas } \\
\text { las preguntas. }\end{array}$ & & & & \\
\hline 18 & Tiene dificultades para aguardar su turno. & & & & \\
\hline 19 & $\begin{array}{l}\text { Interrumpe o se inmiscuye en las actividades de otros } \\
\text { (p. ej. se entromete en conversaciones o juegos). }\end{array}$ & & & & \\
\hline 20 & $\begin{array}{l}\text { Tiene dificultad para permanecer sentado, quedarse } \\
\text { quieto o inhibir impulsos en la clase o en el hogar. }\end{array}$ & & & & \\
\hline
\end{tabular}

Fuente: elaborado por los autores con base en las referencias 6 a 10.

a SNAP: cuestionario para detectar TDAH cuyo nombre deriva de sus creadores Swanson, Nolan y Pelham.

un rango de severidad de cero a 27 puntos. Estudios epidemiológicos importantes, como el tratamiento multimodal de TDAH del Instituto Nacional de Salud (Estados Unidos), utilizaron el SNAP IV como herramienta de detección y de medición de la efectividad del tratamiento (11).

El objetivo del presente trabajo fue valorar la utilidad de la escala SNAP IV ${ }^{5}$ como instrumento de detección de TDAH en niños argentinos de 4 a 14 años de edad (tal como se hizo en la escala ori-

\footnotetext{
5 Si bien hay una versión posterior del mismo autor (la escala SKAMP) de mayor especificidad porque tiene siete grados de severidad (nada, poca, mínima, moderada, severa, muy severa y máxima), se decidió no utilizarla en este estudio porque al tener tantas categorías arroja resultados poco confiables en poblaciones con niveles de educación no muy altos $(9,12)$.
}

ginal). Adicionalmente, se establecieron los puntajes de corte de acuerdo a la sensibilidad y especificidad en la población para las subescalas de déficit de atención e hiperactividad-impulsividad.

\section{MATERIALES Y MÉTODOS}

Se administró la escala de clasificación de TDAH para padres y maestros SNAP IV en dos escuelas seleccionadas dentro del Gran Buenos Aires, Argentina, donde asistían niños y adolescentes de niveles socioeconómicos acordes con la población general según las estadísticas del Instituto Nacional de Estadísticas y Censos (13) (cuadro 1). Se incluyó a todos los alumnos de 4 a 14 años pertenecientes a un total de 30 clases. Participaron 1230 niños y adolescentes (52,3\% varones y $47,7 \%$ mujeres) con un prome- 
CUADRO 2. Características de niños y adolescentes estudiados en dos escuelas del Gran Buenos Aires, para detectar trastornos por déficit de atención con hiperactividad (TDAH), Argentina, 2003

\begin{tabular}{lr}
\hline \multicolumn{1}{c}{ Característica } & \multicolumn{1}{c}{ No. } \\
\hline Estudiantes evaluados con SNAP IVa & 1230 \\
$\begin{array}{l}\text { Edad población estudiada (años): } \\
\quad \text { media (rango) }\end{array}$ & $8,8(4-14)$ \\
Casos SNAP IV positivos & 98 \\
Casos SNAP IV negativos & 1132 \\
$\begin{array}{l}\text { Estudiantes con SNAP y criterios } \\
\quad \text { línicos del DSM IVb positivos }\end{array}$ & $49 / 1230(4 \%)$ \\
$\begin{array}{l}\text { Edad población con resultado } \\
\quad \text { positivo (años): media (rango) }\end{array}$ & $7,9(4-14)$ \\
Relación hombre/mujer en casos & $3,11 / 1$ \\
$\quad$ positivos & \\
\hline
\end{tabular}

Fuente: elaborado por los autores.

a SNAP: cuestionario para detectar TDAH cuyo nombre deriva de sus creadores Swanson, Nolan y Pelham.

b DSM: siglas en inglés para el Manual diagnóstico y estadístico de los trastornos mentales.

dio de edad de 8,8 años, evaluados durante el período escolar de 2003. Se excluyó a los alumnos en educación especial o con otros problemas que impedían la escolaridad común. Los docentes completaron los cuestionarios de los síntomas de cada alumno en su aula - entre 20 y 30 niños por clase.

Luego se seleccionaron todos los cuestionarios de los casos positivos y la misma cantidad de casos negativos apareados por edad y sexo. Se citó a los padres de estos niños a una entrevista clínica en la que además cada uno completó el cuestionario SNAP IV con objeto de determinar los síntomas de sus hijos también en el hogar. Previo a las entrevistas, se solicitó a los padres completar y suscribir un consentimiento con información sobre los objetivos y procedimientos, así como sobre el carácter confidencial de todas las respuestas obtenidas. El estudio fue aprobado por el Comité de Investigación del Hospital Universitario Austral.

Se contó con un equipo de psicopedagogas entrenadas, quienes ayudaron a los docentes y los padres a completar los consentimientos y resolver dudas durante las encuestas.

Las entrevistas clínicas fueron realizadas en persona con la investigadora principal del proyecto, junto con un psiquiatra, una neuropsicóloga y/o las psicopedagogas participantes. Se indagaron antecedentes clínicos e información demográfica, se completó la encuesta SNAP IV por los padres y los criterios diagnósticos del DSM IV según los síntomas clínicos. Los evaluadores realizaron las entrevistas clínicas a los pacientes y controles citados a ciego de los resultados que habían arrojado con la escala SNAP IV. Se consideraron positivos los niños que reunían de seis a nueve criterios diagnósticos para déficit de atención y/o de seis a nueve para hiperactividadimpulsividad según el DSM IV, ya sea bajo la forma por déficit de atención o las formas mixtas con déficit de atención e hiperactividad e impulsividad.

Se analizaron los puntajes de las subescalas para déficit de atención e hiperactividad-impulsividad. Cada subescala consistió en 10 preguntas que describían los criterios del DSM-IV - las 1 a 9 sobre el dominio atencional y las 11 a 19 sobre hiperactividad-impulsividad. Hubo además una pregunta general sobre los síntomas para cada subescala (preguntas 10 y 20). Según la escala original se sumaron las puntuaciones, que tienen un rango de 0 a 3 . La suma, en un rango de 0 a 27 puntos para cada subescala, se dividió por 9 y así se obtuvieron los índices para las subescalas de déficit de atención e hiperactividad-impulsividad. Los datos fueron procesados con una base STAT-A, determinándose la especificidad y sensibilidad, así como los puntajes de corte de acuerdo al percentil 95, la razón pulmón/cabeza (RPC), la razón de probabilidades (RP) y radio Ji cuadrado para encuestas de detección.

\section{RESULTADOS}

Se detectó un total de 98 casos positivos según la escala SNAP IV. Se citaron igual número de casos negativos, apareados por edad y sexo con los casos posi-

FIGURA 1. Distribución de casos positivos de trastornos por déficit de atención con hiperactividad, por edad, Gran Buenos Aires, Argentina, 2003

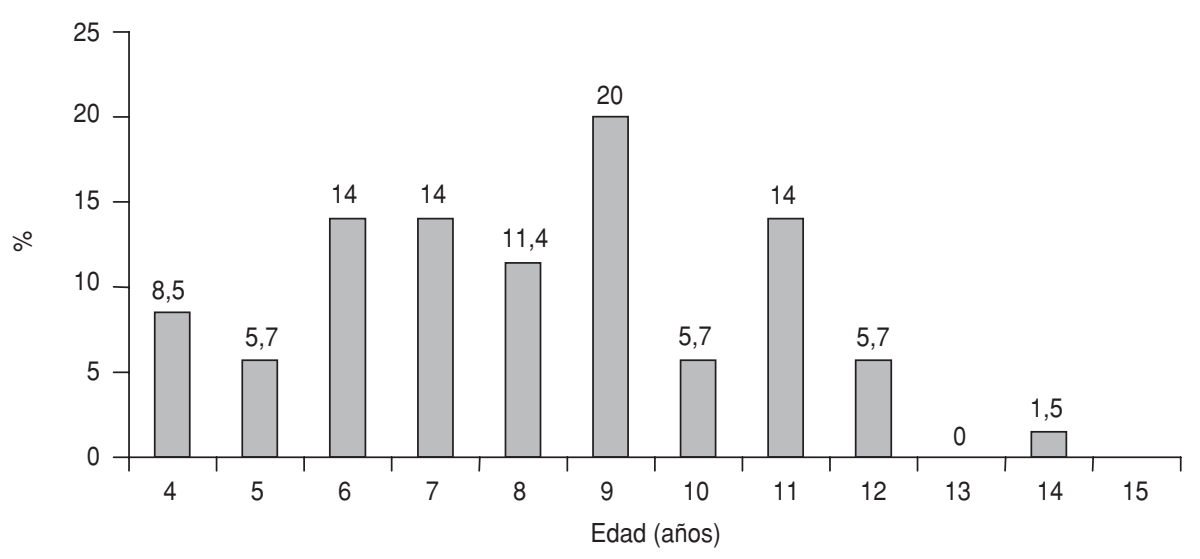

Fuente: elaborado por los autores. tivos, de los cuales solo 94 niños concurrieron a la entrevista clínica. Se obtuvo un grupo de 192 pacientes cuyos padres completaron una entrevista para comparar con los criterios clínicos del DSM IV (cuadro 2). De los 192 niños evaluados, el diagnóstico se confirmó en 49, de los cuales 48 pertenecían al grupo con detección positiva y tenían una edad promedio de 7,9 años (figura 1).

La determinación de la validez empírica para déficit de atención arrojó una sensibilidad de $54 \%$ y una especificidad de $78 \%$, mientras que para hiperactividad-impulsividad estos valores fueron de $86,0 \%$ y $73,5 \%$, respectivamente (cuadro 3). Con estos resultados se determinaron los puntajes de corte para establecer la validez discriminante; se compararon los resultados según los métodos por el percentil 95, la RPC y la razón Ji cuadrado. La mejor relación para déficit de atención se halló con el percentil 95 y el Ji cuadrado. Para hiperactividad-impulsividad coincidieron los tres métodos, los tres con sensibilidad y especificidad significativas.

El puntaje de corte de la encuesta para docentes dio un índice de 1,66 (15/27 puntos) para la subescala de atención y de 1,77 (16/27 puntos) para la subescala de hiperactividad-impulsividad (cuadro 3). Debe destacarse que si bien $7,9 \%$ del total de niños dieron positivo según la escala de sospecha, solo $4 \%$ fueron confirmados conforme a los criterios clínicos para TDAH. Aun así, los casos restantes con criterios de sospecha positivos presentaban otras dificultades en el desempeño escolar y conductual que requerían continuar con evaluaciones diagnósticas. 
CUADRO 3. Puntos de corte de las subescalas de déficit de atención y de hiperactividadimpulsividad, según los métodos estadísticos percentil 95, la razón pulmón/cabeza (RPC) y la razón de Ji cuadrado, Buenos Aires, Argentina, 2003

\begin{tabular}{lcccc}
\hline \multicolumn{1}{c}{ Método por subescala } & $\begin{array}{c}\text { Puntaje } \\
\text { de corte } \\
\text { total }\end{array}$ & $\begin{array}{c}\text { Puntaje } \\
\text { índice de } \\
\text { corte }\end{array}$ & Sensibilidad & Especificidad \\
\hline Atención (rango) & $0-27$ & & & \\
$\quad$ Percentil 95 & $15 / 27$ & $1,66(15 / 27)$ & 72,7 & 65,3 \\
Razón RPC & $18 / 27$ & 2 & 54,5 & 78,8 \\
$\quad$ Razón Ji cuadrado & $15 / 27$ & 1,66 & 72 & 65 \\
Hiperactividad (rango) & $0-27$ & & 86,4 & 73,5 \\
$\quad$ RPC, Ji cuadrado y percentil 95 & $16 / 27$ & $1,77(16 / 27)$ & 86,4 & \\
\hline
\end{tabular}

Fuente: elaborado por los autores.

En cuanto a las características sociodemográficas y el nivel educativo de los padres, se halló que $64 \%$ tenía educación primaria, $27 \%$ educación secundaria, $7 \%$ educación universitaria y en menos de $2 \%$ de los casos uno o ambos padres eran analfabetos. Estos niveles fueron acordes a la distribución poblacional estimada por el Instituto Nacional de Estadísticas y Censos (INDEC).

\section{DISCUSIÓN}

La idea de adaptar una escala de detección de trastornos de conducta a la población local estuvo basada en la necesidad de identificar los síntomas de alarma en poblaciones con riesgo de TDAH, para luego completar el diagnóstico con instrumentos adecuados. También se procuraba facilitar estudios poblacionales escolares, valorar la respuesta a tratamientos según la evolución de los síntomas y contribuir al establecimiento de objetivos terapéuticos.

La escala SNAP IV, desarrollada por Swanson, Nolan y Pelham a partir de la entrevista diagnóstica para niños (DISC-R, por sus siglas en inglés), tiene buena confiabilidad entre encuestadores no entrenados como docentes y padres; también tiene una mayor estabilidad en evaluaciones longitudinales (14-16). En el caso del presente estudio, SNAP IV fue elegida porque, dado su propósito de determinar puntajes de corte acordes con la población de Argentina, esta escala i) se basa estrictamente en los criterios del DSM IV, ii) es fácil de puntuar e interpretar, iii) tiene buena confiabilidad inter-evaluadora en su versión original, iv) es económica y de libre acceso en Internet, v) no requiere de un entrenamiento exhaustivo para su uso y vi) ha sido muy útil en estudios de seguimiento longitudinal de pacientes $(11,17)$. Se eli- gió la escala para maestros dado que en poblaciones latinas habitualmente los puntajes de los padres son más altos y reducen la fiabilidad de la muestra (18). Dicho lo anterior, no obstante, por ser una herramienta de detección no tiene propiedades psicométricas suficientes para ser utilizada en forma aislada como única prueba diagnóstica.

Se pretendió además adaptar la escala a una población con diferentes características a las de los países sajones y con otros patrones culturales de comportamiento, como se ha observado en algunos estudios poblacionales de América Latina (19-25). Como toda escala de detección, no implica una certificación, sino que permite detectar los individuos que requieren completar el diagnóstico (19). Se compararon los criterios de esta escala con los criterios clínicos del DSM IV para determinar la sensibilidad y especificidad, como se realizó en otros trabajos en poblaciones latinas $(24,25)$.

Si bien las escalas de detección o screening por definición tienen alta sensibilidad y baja especificidad, al ser aplicadas por el docente podrían permitirle referir al niño a una evaluación. Los valores de prevalencia en las escalas de despistaje suelen ser altos y solo alrededor de $50 \%$ de los pacientes positivos en los screening resultarían verdaderos positivos $(26,27)$, como ocurrió en la muestra de este estudio, donde solamente la mitad de los casos positivos sospechados terminaron siendo confirmados con los criterios clínicos para TDAH. Sin embargo, en la mitad restante con criterios de alarma se hallaron otras dificultades en el desempeño escolar y conductual, por lo que resultó útil haberlos detectado para realizar una evaluación posterior.

Aunque los puntajes de corte se establecieron tanto con percentil 95 como con RPC y Ji cuadrado, fue preferible tomar en cuenta también los últimos, dado que la población con TDAH no sigue una distribución gaussiana sino en Ji cuadrado $(6,27)$. Asimismo, el hecho de que los puntajes de corte resultaran más bajos que los de la población sajona podría deberse a que la hiperactividad y la mayor distractibilidad son culturalmente aceptados por los padres latinos. Por ejemplo, el caso falso negativo en el screening era de una niña que fue informada con atención normal por su maestra, pese a que recibía soporte pedagógico regular por sus dificultades atencionales y luego mostró franca mejoría en el desempeño escolar con tratamiento.

Otro aspecto fundamental de este estudio radicó en que la mayor parte de la población evaluada no había accedido a diagnóstico ni tratamiento farmacológico previamente, incluyéndose niños desde los 4 años de edad, ya que el TDAH es una preocupación creciente en preescolares (28).

Nuestros hallazgos coincidieron con los mismos subtipos incluidos en el DSM IV, con los de otras poblaciones sudamericanas, como la de Rohde y colaboradores en Brasil, y con los subtipos del mayor estudio estadounidense de seguimiento, el "estudio multimodal de tratamiento de niños con TDAH" (conocido como MTA, por sus siglas en inglés) $(1,11,29)$.

La edad promedio del grupo fue de 7,9 años, con una razón varón/mujer de $3,11 / 1$. Uno de los pocos estudios locales halló una razón varón/mujer diferente, pero fue desarrollado con los criterios del DSM III R y solo se hizo el screening y no la entrevista clínica de diagnóstico (30). Aparte de los datos del presente estudio, que fueron presentados en un congreso, no se encontró otra adaptación en población argentina (31).

Es de esperar que uno de los aportes de esta escala resida en establecer puntajes de corte para la población argentina que ayudarán a realizar estudios epidemiológicos próximos a la idiosincrasia de la población latina.

En cuanto a las limitaciones del estudio hay que mencionar que, si bien hubiera sido preferible establecer puntajes de corte por edad, se optó por respetar la estructura metodológica de la validación de la escala original aun cuando el comportamiento es muy distinto entre un preescolar y un escolar. De hecho estas son limitaciones propias de los criterios del DSM IV para preescolares, como lo 
reflejan estudios de revisión y metaanálisis, en particular porque la hiperactividad excesiva no siempre persiste en la etapa escolar y el déficit de atención es poco consistente para las tareas que realiza un niño pequeño (32). Sin embargo, los puntajes de corte de las escalas de detección en preescolares han resultado confiables tanto en la presente adaptación como en la versión original en inglés, cuya validez ha sido verificada también con otras escalas de construcción similar para este grupo etario $(17,33)$.

Además se respetó el formato original, incluso cuando los criterios del DSM IV en su traducción literal no son tan claros como en la versión en inglés. Por otro lado, aun sabiendo que las escalas de screening de conductas tienen menor sensibilidad y especificidad con respecto a los tests psicométricos, se seleccionó la escala SNAP por su utilidad para estudios de pesquisa y de seguimiento, tal como informan los trabajos del MTA (11). Más todavía, esta escala brinda una mejor relación sensibilidad/especificidad respecto de la escala de Conners, que suele ser la de referencia.

En suma, se encontró que la escala SNAP IV destinada a docentes, con los criterios de corte establecidos, es útil para la detección de TDAH en la población argentina. Los valores de corte para la subescala de atención alcanzaron un índice de 1,66 (15/27 puntos) y para la subescala de hiperactividad-impulsividad de 1,77 (16/27 puntos). Estos puntajes demostraron tener la mejor relación entre sensibilidad y especificidad para la población estudiada (31).

La diferencia en los puntajes de corte hallados en este grupo con respecto a los originales llevan a plantear la necesidad de adaptar las escalas de screening o detección en las poblaciones donde se van a utilizar, en razón de las diferencias culturales y las características particulares de los niños.

Asumir resultados a partir de una encuesta con valores de corte validados puede arrojar conclusiones más exactas, tanto en la detección como en el seguimiento clínico y epidemiológico de la población latina.

Agradecimiento. Los autores agradecen a Marina Drake, especialista en neuropsicología, por su contribución profesional y humana permanente durante la realización del presente estudio, así como a los laboratorios Elly-Lilly por cubrir gastos de materiales y procesamiento estadístico.

\section{REFERENCIAS}

1. American Psychiatric Association. Diagnostic and Statistical Manual of Mental Diseases. $4 .^{\text {a }}$ ed (DSM-IV). Washington, DC: American Psychiatric Publishing; 1994.

2. Barkley R. Chapter I: History. In: Barkley R, ed. Attention-Deficit Hyperactivity Disorder. 3. ${ }^{\mathrm{a}}$ ed. A Handbook for Diagnosis and Treatment. New York: Guillford Publishers; 2006. Pp. 3-52.

3. Posner M, Rothbart M. Research on Attention Networks as a Model for the Integration of Psychological Science. Annu Rev Psychol. 2007;58:1-23.

4. Conners C. Rating scales in attention deficit/ hyperactivity disorder. J Clin Psychiatry. 1998;(59 supl):24-30.

5. Green M, Wong M, Atkins D, Taylor J, Feinleib M. Diagnosis of Attention Deficit/ Hyperactivity Disorder. Technical Review 3 (AHCPR publication 99-0050). Rockville, MD: US. Department of Health and Human Services, Agency for Health Care Policy and Research; 1999.

6. Swanson J. School-based assessments and intervention for ADD students. Irvine, CA: K.C. Publishing; 1992.

7. Pliszka S, Carlson C, Swanson J. Behavioral assessment. In: ADHD with comorbid disorders. Clinical assessment and management. NY: Guilford Press; 1999. Pp. 25-38.

8. Swanson J, Sandman C, Deutsch C, Baren M. Methylphenidate hydrochloride given with or before breakfast: I. Behavioral, cognitive and electrophysiologic effects. Pediatrics. 1983;72: 49-55.

9. Murray DW, Bussing R, Fernandez M, Wei Hou, Garvan CW, Swanson JM, et al. Psychometric properties of teacher SKAMP ratings from a community sample. Assessment. 2009;16(2):193-208.
10. Swanson J, Sergeant J, Taylor E, Sonuga-Barke E, Jensen P, Cantwell D. Trastorno por déficit de atención con hiperactividad y trastorno hipercinético. Lancet. 1998;32(6):360-5.

11. MTA Cooperative Group. National Institute of Mental Health Multimodal Treatment Study of ADHD Follow-up: 24-Month Outcomes of Treatment Strategies for AttentionDeficit/Hyperactivity Disorder. Pediatrics. 2004;113(4):754-61.

12. Hernández Sampieri R, Fernández-Collado C, Baptista LP. Metodología de la investigación. 4.a ed. Mexico: Mc Graw Hill Edit; 2006.

13. Instituto Nacional de Estadística y Censos (INDEC), Argentina. Censo Poblacional Nacional 2003. Disponible en: http//:www. indec.gov.ar. Acceso el 03 de abril de 2011.

14. Shaffer D, Schwab-Stone M, Fisher P, Cohen P, Piacentini J, Davies M, et al. The diagnostic interview schedule for children-revised version (DISC-R): I. preparation, field testing, interrater reliability, and acceptability. J Am Acad Child Adolesc Psychiatry. 1993;32: 643-50.

15. Bussing R, Fernandez M, Harwood M, Wei Hou, Garvan CW, Eyberg SM, et al. Parent and teacher SNAP-IV ratings of attention deficit hyperactivity disorder symptoms: psychometric properties and normative ratings from a school district sample. Assessment. 2008;15(3):317-28.

16. Collett B, Ohan J, Myers K. Ten-year review of rating scales. V: scales assessing attentiondeficit/hyperactivity disorder. J Am Acad Child Adolesc Psychiatry. 2003;42:1015-37.

17. Swanson J, Schuck S, Mann M, Carlson C, Hartman K, Sergeant J, et al. Categorical and Dimensional Definitions and Evaluations of Symptoms of ADHD: The SNAP and the SWAN Ratings Scales. Disponible en: http//:www.adhd.net . Acceso el 04 de abril de 2011.

18. Servera M, Cardo E. ADHD Rating Scale-IV en una muestra escolar española: datos normativos y consistencia interna para maestros, padres y madres. Rev Neurol. 2007;45:393-9.

19. Pineda D, Henao G, Puerta I, Mejía S, Ardila A, Roselli M, et al. Uso de un cuestionario breve en español basado en los criterios del DSM IV para el diagnóstico de deficiencia atencional. Rev Neurol. 1999a;28:365-72.

20. Pineda D, Kamphaus R, Mora O, Puerta I, Palacio L, Jiménez I, et al. Uso de una escala multidimensional para padres de niños de 6 a 11 años en el diagnóstico de deficiencia atencional con hiperactividad. Rev Neurol. 1999; 28:952-9.

21. Urzúa A, Domic M, Ramos M, Cerda A, Quiroz J. Propiedades psicométricas de tres escalas de evaluación del trastorno por déficit de atención con hiperactividad en escolares chilenos. Rev Panam Salud Publica. 2010; 27(3):157-67.

22. Farré Riva A, Narbona J. Escala de Conners en la evaluación del trastorno por déficit de atención con hiperactividad. Nuevo estudio de validación y análisis factorial en niños españoles. Rev Neurol. 1997;25:200-4.

23. Cornelio-Nieto JO, Borbolla-Sala M, GarcíaValdovinos S. Estudio poblacional de diagnóstico comparativo entre el DSM-IV y el cuestionario para escolares y adolescentes latinoamericanos para la detección del trastorno por déficit de atención/hiperactividad. Rev Neurol. 2010;50(3 supl):S119-23.

24. Montiel-Nava C, Peña J, López M, Salas M, Zurga J, Montiel-Barbero I, et al. Estimaciones de la prevalencia del trastorno por déficit de atención-hiperactividad en niños marabinos. Rev Neurol. 2002;35:1019-24. 
25. Sánchez C, Ramos C, Díaz F, Simón M. Validación de la escala de evaluación del trastorno por déficit de atención/hiperactividad (EDAH) en población adolescente. Rev Neurol. 2010; 50:283-90.

26. Snyder S, Cornwell S, Quintana H. Review of clinical validation of ADHD behavior rating scales. Psychol Rep. 2006;99:363-78.

27. Shur-Fen Gau S, Lin C, Hu F, Shang C, Swanson J, et al. Psychometric Properties of the Chinese Version of the Swanson, Nolan, and Pelham, Version IV Scale-Teacher Form. J Pediatr Psychol. 2009;34(8):850-61.

28. Vaquerizo-Madrid J. Evaluación clínica del trastorno por déficit de atención/hiperactivi- dad, modelo de entrevista y controversias. Rev Neurol. 2008; 46 (1 supl):S37-41.

29. Rohde L. ADHD in Brazil: the DSM IV Criteria in a culturally different population. J Am Acad Child Adolesc Psychiatry. 2002; 41(9):1131-3.

30. Michanie C, Marquez M, Estevez P, Steimberg L. Trastorno por déficit de atención con hiperactividad. Arch Argent Pediatr. 1993;91:231-8.

31. Grañana N, Richaudeau A, Robles Gorriti C, Scotti ME, Fejerman N, Allegri R. Detección de síntomas para trastorno por déficit de atención e hiperactividad: escala SNAP IVValidación en Argentina. Revista Neurologica Argentina. 2006;28(1 supl):20.
32. Dreyer B. The diagnosis and management of attention-deficit/hyperactivity disorder in preschool children: the state of our knowledge and practice. Curr Probl Pediatr Adolesc Health Care. 2006;36:6-30.

33. DuPaul GJ, Power TJ, Anastopoulos AD, Reid R. Manual for the AD/HD Rating Scale-IV. New York: Guilford Press; 1998

Manuscrito recibido el 26 de junio de 2010. Aceptado para publicación, tras revisión, el 8 de febrero de 2011.

ABSTRACT Objective. Assess the usefulness of the SNAP-IV scale as an instrument for detecting attention deficit hyperactivity disorder (ADHD) in Argentine children aged 4 to 14 years.

Assessment of attention Methods. The SNAP-IV scale was adapted and administered to a group of 1230 deficit hyperactivity: SNAP-IV scale adapted to Argentina schoolchildren in the province of Buenos Aires, Argentina. The diagnosis was determined with the clinical control, based on the criteria of the Diagnostic and Statistical Manual of Mental Disorders, 4th edition. The sensitivity and specificity, as well as the cut-off scores for the SNAP-IV scale in the population studied, were determined.

Results. The score on the SNAP-IV scale with the best correlation between sensitivity and specificity was established in order to determine the true positive cases that in fact had a clinical diagnosis. The cut-off scores obtained were: 1.66 (15/27 points) for the attention deficit subscale and 1.77 (16/27 points) for hyperactivity/impulsivity in the population studied.

Conclusions. The SNAP-IV scale for detection of ADHD is considered to be valid in the population studied as long as the cut-off scores are modified to obtain the best sensitivity/specificity ratio based on the cultural and socioeconomic features of the population.

Key words Attention deficit disorder with hyperactivity; mental health; Argentina. 